\title{
M. Fin, H. Steenwijk (a cura di), Gerasim Zelić e il suo tempo, Firenze University Press, Firenze 2019, pp. I25.
}

Il volume Gerasim Zelić e il suo tempo è agile e - mi verrebbe da dire - dilettevole. Mi permetto di usare questo aggettivo nonostante si tratti di un libro pubblicato da un editore scientifico come la Firenze University Press (<https://media.fupress.com/files/pdf/24/3998/3998_21706>), frutto di una ricerca biennale intitolata The Interconfessional Polemic Between the Ortodox Serbs and Catholic Church in the Manuscripts of Gerasim Zelic, finanziata dal Dipartimento di Studi Linguistici e Letterari dell' Università degli Studi di Padova. I contributi presentati riguardano un periodo storico poco noto e sono focalizzati su un personaggio non molto studiato. Il volume, curato da Monica Fin e Han Steenwijk, contiene nove saggi che si leggono con grande interesse.

Nel contributo intitolato La polemica confessionale fra ortodossi e cattolici negli scritti di Gera$\operatorname{sim}$ Zelićla curatrice sottolinea che scannerizzando "circa 300 documenti, per un totale di oltre 1000 carte manoscritte redatte in serbo, italiano, tedesco, greco e latino, è stato ricavato un corpus finale costituito da 140 documenti strettamente pertinenti alla tematica indagata del progetto. I manoscritti scelti coprono un arco di tempo che va dal gennaio 1794 al settembre 1830 . La maggior parte del corpus è costituita dalla corrispondenza personale di Gerasim Zelić, oltre a numerosi documenti ufficiali emanati dalle autorità veneziane, austriache e francesi che all'epoca si avvicendarono nella dominazione della Dalmazia" (p. 3).

Il materiale digitalizzato è disponibile in linea ed è consultabile all'indirizzo: <http://www. maldura.unipd.it/zelic/> il che consente a tutti di usufruire dei risultati di questa ricerca. "Oltre che dal punto di vista storico-culturale - scrive ancora la Fin - i documenti raccolti costituiscono materiale di grande rilievo anche per l'analisi di tipo linguistico: a tale proposito, particolare interesse è già stato espresso dal comitato scientifico del Rečnik slavenosppskog jezika, che ha individuato nei manoscritti legati alla figura di Zelić un valido corpus di riferimento per le ricerche sulla lingua serba di epoca precedente alla standardizzazione" (p. s).

Per rendere il materiale accessibile anche a chi non conosce l'alfabeto cirillico si è deciso di traslitterare i testi in caratteri latini. Bisogna ammettere che il livello tecnologico conseguito dai collaboratori di questo progetto si avvicina - rispetto a quanto si sarebbe potuto realizzare in era pre-elettronica - al puro Science Fiction visto il risultato ottenuto!

Si può presumere che l'altro curatore, Han Steenwijk, sia 'l'anima' della parte tecnologica del progetto perché nel suo contributo intitolato Lingue letterarie storiche in ambito culturale serbo: alcuni problemi di codifica HTML dimostra come un linguista possa usare questi risultati tecnologica- 
mente così avanzati: grazie a una lettera presente in questo corpus (inviata da Stratimirović a Zelić nel I826) che di solito si considera scritta in 'slavenosrpski, l'autore individua le proporzioni precise di diversi strati linguistici, che vanno dallo slavo ecclesiastico al russo e infine anche al serbo, cioè a qualche traccia della lingua parlata dell'epoca.

Questi due interventi danno un quadro dei risultati del progetto relativamente all'aspetto filologico del materiale fino ad oggi in gran parte inedito e poco studiato. Gli altri sette contributi si occupano del periodo storico e trattano anche temi non collegati all'argomento specifico del progetto: ampliando lo sguardo a una visuale più ampia, che non riguarda solo il difficile rapporto tra le due religioni cristiane descritto nell'autobiografia di Gerasim Zelić, questi scritti aprono nuovi orizzonti di natura storica, filologica e sociale e suggeriscono di fatto quali possano essere nuove problematiche ancora da studiare.

Egidio Ivetić, anche lui come i due curatori docente dell' Università di Padova, ottimo conoscitore della storia dei mondi che circondano l'Adriatico, propone una serie di questioni e tematiche che s' intrecciano sulla sponda orientale dell'Adriatico e mostra quanto fosse complessa la realtà della Dalmazia all'epoca di Zelić. Nel suo contributo intitolato Adriatico, come sfondo enumera una serie di tratti specifici, problemi di natura economica e sociale, ma sottolinea soprattutto il fatto che "questo litorale sia stato sempre un confine, il limite di qualcosa. Una situazione determinata da quel netto distacco morfologico che separa la costa dall'interno, gli uomini del litorale rispetto a quelli dell'interno; un distacco a tratti marcato, completamente diverso rispetto a quanto avveniva lungo l'arco delle lagune venete. [...] La storia di questa regione è soprattutto la storia degli innumerevoli piccoli e grandi confini, che meritano di essere pazientemente censiti e analizzati” (p. 48).

Il contributo di un altro storico, Drago Roksandić, professore emerito dell'Università di Zagabria, sembra voler rispondere proprio a questa richiesta. In un saggio dal titolo Gerasim Zelić - Homo Mediterraneus? Roksandić segue i viaggi dell'ecclesiastico illuminato' descritti nella sua opera autobiografica Žitije. A causa del "netto distacco morfologico che separa la costa dall'interno" (p. 48) cui fa cenno Ivetić, Roksandić giustamente descrive il forte contrasto tra il monaco e la sua gente: Zelić metteva in evidenza tutte le affinità (raramente anche le emozioni) che riscontrava nel confronto con le grandi città mediterranee (Venezia, Trieste, Zara, Napoli, Bari...), sebbene provenisse da un piccolo villaggio dell'entroterra dalmata e svolgesse il suo servizio religioso presso un monastero "nel deserto" montagnoso. Noi oggi possiamo capire perché Zelić era "perennemente assetato di nuovi modelli di pensiero e di comportamento, urbani, dotti, civilizzati” (p. 99), ma per i suoi conterranei "egli divenne sempre di più un estraneo per loro, e ciò malgrado fosse proprio di loro che aveva più bisogno" (ibidem).

Anche Dorota Gil dell'Università di Cracovia presenta un contributo relativo a un diverso ambito, contributo che viene pubblicato, così come gli scritti di Grdinic e Vukašinovic, in serbo. Da anni, Dorota Gil studia le problematiche relative ai rapporti religiosi tra gli slavi, non solo per quel che concerne le difficoltà e le tensioni fra ortodossi e cattolici, ma trattando anche la componente protestante. Nel contributo intitolato Srbi u I8. veku prema katolicizmu i protestantizmu - mehanizmi i strategije asimilacije novih kulturnih elemenata, con grande maestria e in modo convincente, mostra come i futuri intellettuali serbi frequentavano più spesso le università straniere dei paesi in cui si professava la religione protestante piuttosto che quelle dei paesi cattolici. Gil riporta il numero degli iscritti che sono davvero alti: solo a Bratislava vi erano 80 studenti serbi nella prima metà del Settecento, che nel periodo tra il 1730-1830 salgono a 670! 
Per quanto riguarda gli altri due contributi pubblicati in lingua serba, va rilevato che quello di Vladimir Vukašinović, professore della Facoltà di teologia di Belgrado, (Konfesionalno-liturgičke polemike u teološkim spisima Zelićevih savremenika) tratta un altro tema legato alla religione, dimostrando che la maggior parte dei testi teologici dell'epoca di Zelić erano polemici, e non solo nei confronti dei cattolici. Questo stesso tono si nota addirittura nella lirica e come esempio viene citata una nota poesia di Zaharije Orfelin, contemporaneo di Zelić.

Il titolo del contributo di Nikola Grdinić, professore dell' Università di Novi Sad, Avanture $i$ avanturisti u Zelićevom Žitiju promette molto. Ritengo, però, che in questo caso Grdinić abbia voluto provocare i futuri ricercatori, sfidandoli ad approfondire il valore delle avventure e degli avventurieri nell'autobiografia di Zelić. Se è accettabile classificare senza problemi come avventurieri Simeon Ivković e Venedikt Kraljević, mi pare che sia molto complicato inserire Zelić in questa categoria. Prima di tutto: è difficile credere che questo monaco illuminato e sempre in cerca di nuovi saperi sia stato un 'imprenditore' e che le sue gesta manifestatesi viaggiando in molti paesi lontani fossero motivate non solo oggettivamente ma anche intimamente dal desiderio di diventare ricco e potente. In primo luogo, lo Žitije ci mostra un monaco ardito che fa di tutto per reperire libri e quanto fosse necessario alla chiesa ortodossa in Dalmazia, e non come una persona che cerca di diventare potente e di ascendere nella gerarchia ecclesiastica. Zelić è spinto dall'entusiasmo di conoscere, di vedere, di imparare, e non di arricchirsi. Se poi chiede e riesce ad ottenere il grado di archimandrita dal patriarca bizantino a Istanbul, lo fa perché in Dalmazia mancava chi potesse assegnargli questo titolo, che poteva risultare vantaggioso per il suo popolo privo di autorità ecclesiastiche e di ogni tipo di istruzione. Non c'è dubbio che Zelić fosse consapevole del proprio spessore intellettuale a paragone di quello degli ecclesiastici che lo circondavano. Sa di valere più degli altri perché ha visto e imparato tanto viaggiando per anni. Era questo il suo capitale. Ma attribuirgli l'etichetta di avventuriero che si voleva arricchire è un'accusa che Grdinić dovrebbe argomentare meglio. O forse bisogna leggere il suo saggio come una provocazione che vuole spingere futuri ricercatori a approfondire la condizione economica non solo di Zelić, ma anche di tutto l'ambiente ecclesiastico dell'epoca.

Messi a confronto i due contributi scritti dalle colleghe che insegnano all'Università di Pescara, Persida Lazarević Di Giacomo e Maria Rita Leto, sono di stoffa diversa, di orientamento differente, ma tutti e due di enorme importanza e di grande interesse. Fu colpa del 1817 : l'uscita di Solarić dallo Žitije di Zelić è il titolo del contributo di Persida Lazarević Di Giacomo. Un testo estremamente preciso, filologico nel senso più alto del termine, in cui l'autrice si occupa della mancata pubblicazione dello Žitije di Zelić da parte della tipografia Teodosio di Venezia. Persida Lazarević Di Giacomo dimostra, attraverso un'attenta analisi di una serie di lettere e di altri documenti che, in quel fatidico 1817 , furono proprio gli innumerevoli impegni di Solarić ad aver impedito la pubblicazione del volume, nonostante lo stesso Solarić avesse già ricevuto da Zelić del denaro per coprire una parte delle spese di pubblicazione. Particolarmente vivida è la descrizione dei difficili rapporti tra l'autore dell'autobiografia e l'editore, sì volenteroso ma evidentemente non predestinato a pubblicare questo manoscritto. La trattazione è ben ponderata, convincente, tanto da risultare un lavoro di spicco nell'ambito degli studi sulla cultura serba.

L'altro contributo, proposto da Maria Rita Leto, intitolato Due autobiografie a confronto: Život i priključenije di Dositej Obradović e Žitije di Gerasim Zelić sarà sicuramente di grande aiuto agli studenti di slavistica e a tutti i lettori interessati a conoscere i contenuti, le somiglianze e le differenze tra le autobiografie di queste due personalità quasi coetanee. 
Di rado succede che i risultati di una ricerca restino come fondamenta per future indagini e al tempo stesso aprano la strada a nuovi orizzonti e ammoniscano che il periodo oggetto d'interesse sia una miniera di temi ancora da studiare. Non c'è dubbio che le problematiche legate allo spazio e al tempo qui presentate siano complesse: merito principale di questo volume è di suggerire delle direttrici sulle quali bisogna ancora tornare con nuove ricerche.

Marija Mitrović 